\title{
EVOLUTION OF RADIAL ABUNDANCES GRADIENTS FROM PLANETARY NEBULAE
}

\author{
W.J. MACIEL \\ Instituto Astrônomico e Geofísico da USP, Av. Miguel Stefano 4200, 04301-904 São \\ Paulo SP, Brazil \\ and \\ J.KÖPPEN \\ Inst.f.Theor.Physik u. Sternwarte, Olshausenstr. 40, DW 2300 Kiel, Germany
}

The presence of radial abundance gradients is now well established, both in our Galaxy and in other spirals. These gradients were originally derived from oxygen abundance in HII regions, but in the past few years, other elements produced by massive stars have also been shown to display radial abundance gradients similar to the oxygen gradient, which amounts to $-0.07 \mathrm{dex} \mathrm{kpc}^{-1}$. Moreover, planetary nebulae (PN), belonging to the intermediate disk population (the so-called Peimbert "type II" PN) also exhibit gradients of the same order of magnitude as the HII regions.

The origins of the gradients is not yet clear, although several possibilities have been proposed in the literature, ranging from the adequate gas flow to variations of the SFR/IMF or of the chemical yields. Also connected with the origin of the gradients is the question of their time evolution. In this respect, planetary nebulae are likely to give a significant contribution. Originated from progenitor stars in a large range of main sequence masses $\left(0.8\right.$ to $\left.8 \mathrm{M}_{\odot}\right)$, PN include objects of different populations, whose properties are reflected in their chemical compositions. Therefore, by determining the radial gradients of the main heavy elements in a sample of disk PN, one would be able in principle to study the time evolution of the interstellar medium out of which the PN progenitor stars have been formed.

In this paper, an effort is made to determine radial gradients of the elements $\mathrm{O}, \mathrm{S}, \mathrm{Ne}$ and Ar for PN of types I,II and III, according to the classification scheme originally proposed by Peimbert. The obtained gradients and their variations along the PN sequence are then interpreted in terms of a model for the chemical evolution of the Galaxy.

Work partially supported by CNPq, FAPESP, and DFG (SFB 328) 